\title{
ON THE PERFORMANCE OF UWB AND DS-SPREAD SPECTRUM COMMUNICATION SYSTEMS
}

\author{
Brian M. Sadler, Ananthram Swami
}

Army Research Laboratory, Adelphi, MD 20783, \{bsadler,aswami\}@arl.army.mil

\begin{abstract}
We consider a binary spread spectrum system, with possibly episodic transmission; i.e., the system transmits $n$ pulses per information bit, and allows for off time separation between pulses. Processing gain enables low power transmission, and the ultra-wideband (UWB) spreading limits the interference effects into narrowband systems. We consider the issues of processing gain, jamming margin, coding gain, and multi-user interference (MUI) for a single user matched filter receiver. The introduction of episodic transmission, with a corresponding reduction in bit rate, provides system fle ibility with respect to both MUI rejection and handling multipath channels. Highly episodic transmission provides nearly orthogonal users, even with system asynchrony and no power control. Performance of a single user matched filter is given via a Chernoff bound on bit error rate, in the presence of MUI.
\end{abstract}

\section{CODING GAIN \& JAMMING MARGIN}

We consider a binary spread spectrum system, with possibly episodic transmission; i.e., the system transmits $n$ pulses per information bit, and allows variable off-time separation between pulses. Processing gain enables low power transmission, and the ultrawideband (UWB) spreading limits the interference effects into narrowband systems, e.g., see [1]. A fundamental issue in direct-sequence (DS) CDMA systems is the multi-user interference (MUI), which severely limits single-user matched filter receiver performance, and generally requires strict power control or MUI rejection techniques. The introduction of episodic transmission, with a corresponding reduction in bit rate, provides system fle ibility with respect to both MUI rejection and handling multipath channels. In this paper we focus on the issues of co-channel interference and MUI.

Our analysis in this section follows classical direct sequence (DS) spread spectrum treatments [2], with slight modification to accommodate the episodic aspects. In keeping with direct sequence terminology, we use the terms chip and pulse interchangeably. The chip sequence $b_{i}$ is modeled as random ternary, with $\operatorname{pmf}^{1}$

$$
p_{b}\left(b_{i}\right)=(1-2 \alpha) \delta\left(b_{i}\right)+\alpha \cdot \delta\left(b_{i}-1\right)+\alpha \cdot \delta\left(b_{i}+1\right),
$$

and $0<\alpha \leq 1 / 2$. Thus, in any given chip slot, there is probability $1-2 \alpha$ that no chip occurs, and probability $2 \alpha$ that \pm 1 occurs. The case $\alpha=1 / 2$ corresponds to conventional DS (no episodic transmission). Notice that with the episodic model, transmission of $n$ chips requires (on average) $n /(2 \alpha)$ chip slots. For e ample, with $\alpha=1 / 2$ e actly $n$ slots are needed for transmission of $n$ chips, while with $\alpha=1 / 4,2 n$ slots are now required (with half the slots empty).

Consider an $(n, k)$ block encoding. Let $T_{c}$ be the chip duration, and $W=1 / T_{c}$ be the (appro imate) system bandwidth. Let $T_{b}$ be the bit time, and $R_{b}=$ $1 / T_{b}$ the bit rate. Now, $T_{b}$ is the time required for $n / k$ chips, which is

$$
T_{b}=\frac{n / k}{2 \alpha} T_{c}=\frac{n T_{c}}{2 \alpha k} .
$$

Equation (2) indicates how the coding, system bandwidth, and bit rate are related. In particular, it is possible to increase the system bandwidth (smaller $T_{c}$ ) and concurrently lower $\alpha$ (increase the average time between pulses) to maintain a constant $T_{b}$. As $T_{c}$ becomes appropriately small, the system enters the UWB regime.

Example. Suppose $W=1 \mathrm{GHz}$, and $n=100$ chips per bit (repetition coding, $k=1$ ). From $(2), 10^{9} / 10^{2}=$ $R_{b} / 2 \alpha$. The fastest bit rate occurs with $\alpha=1 / 2$, or $R_{b}=10 \mathrm{Mbps}$. For $\alpha=10^{-4}$, corresponding to e tremely episodic transmission, $R_{b}=2 \mathrm{kbps}$.

Classical DS is also characterized by the bandwidth e pansion factor, $W / R_{b}$. Here,

$$
\frac{W}{R_{b}}=\frac{T_{b}}{T_{c}}=\frac{n}{2 \alpha k} .
$$

\footnotetext{
${ }^{1}$ The model nd $n$ lysis re e sily $d$ pted to the $c$ se of unipol $r$ episodic pulsing, e.g., for PPM.
}

U.S. Government work not protected by U.S. Copyright. 2002 IEEE Conference on Ultra Wideband Systems and Technologies 
In conventional DS $(\alpha=1 / 2)$, we recover $T_{b} / T_{c}=$ $n / k$.

Let $c_{i} \in\{0,1\}$ be the encoded information bit stream. The UWB signal is given by $b_{i}\left(2 c_{i}-1\right) g(t-$ $i T_{c}$ ), where $g(t)$ is the pulse waveform of duration $T_{c}$, and integer $i$ denotes the chip number. We assume that $c_{i}$ and $b_{i}$ are synchronous, so that information is applied only when $b_{i} \neq 0$.

Denote the interference as $z(t)$, and

$$
r_{i}(t)=b_{i}\left(2 c_{i}-1\right) g\left(t-i T_{c}\right)+z(t),
$$

for $i T_{c} \leq t \leq(i+1) T_{c}$. Let $\mathcal{E}_{c}=\int_{0}^{T_{c}}|g(t)|^{2} d t$. The coherent MF receiver is matched to $g(t)$, multiplies by the chip sequence $b_{i}$, and the output is sampled once per chip. The resulting output samples $y_{i}$ will be zero if $b_{i}=0$; when $b_{i} \neq 0$ we obtain

$$
y_{i}=\int_{0}^{T_{c}} r_{i}(t) b_{i} g(t) d t=\left(2 c_{i}-1\right) \mathcal{E}_{c}+b_{i} v_{i},
$$

where the interference contribution is

$$
v_{i}=\int_{0}^{T_{c}} g(t) z\left(t+(i-1) T_{c}\right) d t
$$

The soft decoder computes the correlation metric $C M_{i}=\sum_{j=1}^{n}\left(2 c_{i j}-1\right) y_{j}, i=1,2, \cdots, 2^{k}$, where $c_{i j}=$ $j$ th bit of the $i$ th codeword. (Note that while $y_{i}=0$ for some values of $i$, we avoid cumbersome re-inde ing and simply write $j=1, \cdots, n$ with the understanding that $j$ no longer necessarily corresponds to successive chip intervals.) WLOG, consider the all-zero codeword $c_{1 j}=0, j=1, \cdots, n$. We obtain

$$
C M_{1}=n \mathcal{E}_{c}-\sum_{j=1}^{n} b_{j} v_{j}
$$

while for an arbitrary $m$ th metric $(m \neq 1)$ we find

$$
C M_{m}=n \mathcal{E}_{c}\left(1-\frac{2 \omega_{m}}{n}\right)+\sum_{j=1}^{n}\left(2 c_{m j}-1\right) b_{j} v_{j}
$$

where $\omega_{m}=\sum_{j=1}^{n} c_{m j}$ is the weight of the $m$ th codeword. To determine the probability of a codeword error consider $D=C M_{1}-C M_{m}$,

$$
D=2 \mathcal{E}_{c} \omega_{m}-2 \sum_{j=1}^{n} c_{m j} b_{j} v_{j}=2 \mathcal{E}_{c} \omega_{m}-D_{i n t} .
$$

We invoke the central limit theorem and assume $D_{\text {int }} \sim$ $N\left(0, \sigma_{m}^{2}\right)$ for reasonably large codeword length $n$. Using $E\left[b_{i} b_{j} \mid b_{i}, b_{j} \neq 0\right]=E\left[b_{i}^{2} \mid b_{i} \neq 0\right] \delta_{i j}=\delta_{i j}$, we find

$$
\begin{aligned}
E\left[v_{i}^{2}\right] & =\int_{0}^{T_{c}} \int_{0}^{T_{c}} g(t) g(\tau) \phi_{z}(t-\tau) d t d \tau \\
& =\int_{-\infty}^{\infty}|G(f)|^{2} \Phi_{z}(f) d f
\end{aligned}
$$

where $\phi_{z}(\tau)\left(\Phi_{z}(f)\right)$ is the autocorrelation (power spectrum) of the interference $z(t)$, and

$$
\sigma_{m}^{2}=4 \sum_{j=1}^{n} c_{m j}^{2} E\left[v_{j}^{2}\right]=4 \omega_{m} E\left[v_{j}^{2}\right] .
$$

For spectrally flat interference, $\Phi_{z}(f)=J_{0},|f| \leq$ $W$, where $W=T_{c}^{-1}$ is the UWB channel bandwidth. Now, $\sigma_{m}^{2}=4 \omega_{m}\left(J_{0} \mathcal{E}_{c}\right)$. For $m \neq 1, D \sim N\left(2 \mathcal{E}_{c} \omega_{m}, \sigma_{m}^{2}\right)$, and so the probability of incorrectly choosing the $m$ th codeword is

$$
\operatorname{Pr}(D<0)=\mathcal{Q}\left(\sqrt{\frac{\mathcal{E}_{c} \omega_{m}}{J_{0}}}\right) .
$$

Denoting the bit energy as $\mathcal{E}_{b}$, then $\mathcal{E}_{c}=(k / n) \mathcal{E}_{b}$, and the bound may be rewritten as

$$
\operatorname{Pr}(D<0)=\mathcal{Q}\left(\sqrt{\frac{\mathcal{E}_{b}}{J_{0}} \frac{k}{n} \omega_{m}}\right),
$$

where $\frac{\mathcal{E}_{b}}{J_{0}}$ is the signal-to-interference ratio per bit, and $\frac{k}{n} d_{m i n} \leq \frac{k}{n} \omega_{m}$ is the coding gain. The coding gain depends on $(n, k)$ and $d_{m i n} \leq \omega_{m} \forall m$, and is not directly affected by choice of $\alpha$. A union bound on the total probability of codeword error is then

$$
\operatorname{Pr}(\text { codeword error })=P_{M} \leq \sum_{m=2}^{2^{k}} \mathcal{Q}\left(\sqrt{\frac{\mathcal{E}_{b}}{J_{0}} \frac{k}{n} d_{m i n}}\right)
$$

Now consider a wideband jammer with fi ed average power $J_{a v}$. (A similar analysis may be carried out for narrowband interference.) Then, $J_{a v}=J_{0} W$, where $J_{0}$ is the jammer power spectrum. Let the average signal power be $P_{a v}$, so that $\mathcal{E}_{b}=P_{a v} T_{b}=P_{a v} / R_{b}$. Therefore the bit SNR is

$$
\frac{\mathcal{E}_{b}}{J_{0}}=\frac{P_{a v} / R_{b}}{J_{a v} / W}=\frac{W / R_{b}}{J_{a v} / P_{a v}}
$$

From our earlier discussion, $W$ may be increased while maintaining $R_{b}$ constant by varying $\alpha$. Thus, for a given jamming margin $J_{a v} / P_{a v}$, the processing gain can be increased via an increase in bandwidth. Narrower pulses, combined with episodic transmission, allows an increase in system bandwidth for the same code rate and information rate. (Note that this reasoning is based on the assumption of finite interference power $J_{a v}$, consequently there is no improvement against thermal noise which is assumed to occupy all frequencies and so has infinite power. This case is treated in the ne $t$ section.)

As in the DS case, we may also consider the binary repetition code, corresponding to $k=1$. Now, $(n / k) \omega_{m}=1$ in the above bounds, and performance is enhanced only via the processing gain $W / R_{b}$. 


\section{MF PERFORMANCE WITH MUI}

We consider the matched filter (MF) receiver performance for user $k$, in AWGN with $K$ total users. We assume the users are synchronous in the sense that the chip intervals are assumed to be aligned. However, as we show, the issue is not whether the chip timing is precisely aligned, rather it is how often the episodic chip sequences of other users collide with the desired users sequence. The analysis proceeds along the lines of Viterbi [3], adapted for episodic transmission.

Assume $n$ chips per information bit with repetition coding, and let $\sqrt{\mathcal{E}_{j}}$ denote the energy of the $j$ th user at the receiver. In the $i$ th chip interval the received waveform is

$$
\begin{aligned}
r_{i}(t) & = \\
& \pm \sqrt{\mathcal{E}_{k}} b_{i} g\left(t-i T_{c}\right)+\sum_{\substack{j=1 \\
j \neq k}}^{K} \tilde{b}_{j} \sqrt{\mathcal{E}_{j}} g\left(t-i T_{c}\right)+z(t),
\end{aligned}
$$

denoting the desired user, the MUI, and the AWGN, respectively. Here, $\tilde{b}_{j} \in\{0, \pm 1\}$ models the combined spreading code and data for the $j$ th user, with pmf given by (1), while $b_{i}$ is the spreading sequence for the user of interest ( $k$ th user). For each chip, the receiver performs matched filtering to $g(t)$, multiplies by $b_{i}$, and samples the output, yielding

$$
y_{i}=\int_{0}^{T_{c}} b_{i} r_{i}(t) g(t) d t .
$$

Carrying out the integration we obtain

$$
y_{i}=-\sqrt{\mathcal{E}_{k}}+\sum_{\substack{j=1 \\ j \neq k}}^{K} b_{i} \tilde{b}_{j} \sqrt{\mathcal{E}_{j}}+\tilde{v}_{i},
$$

where we assume WLOG the information bit equals -1 . Note that in (18), $y_{i}=0$ when $b_{i}=0$. As in the previous section, we now assume $y_{i}, i=1, \cdots, n$, is the subsequence conditioned on $b_{i} \neq 0$. The noise term $\tilde{v}_{i}=b_{i} v_{i}$ is conditionally Gaussian when $b_{i} \neq 0$. Also, $x=b_{i} \tilde{b}_{j}$, conditioned on $b_{i} \neq 0$, has the same $\mathrm{pmf}$ as $\tilde{b}_{j}$, given by (1). The decision variable for one information bit is obtained by integrating this $n$-chip subsequence,

$$
y=\sum_{i=1}^{n} y_{i} .
$$

Ne t we develop a Chernoff bound on the probability of bit error (with -1 transmitted), given by

$$
\dot{P}_{e}=\operatorname{Pr}(y>0 \mid-)<\min _{\rho>0} E\left[\operatorname{e} \mathrm{p}\left(\rho \sum_{i=1}^{n} y_{i}\right)\right] \text {. }
$$

Using our iid assumptions, and assuming independence between the spreading sequences and the noise, we can show that

$$
\begin{aligned}
& E\left[\operatorname{e} \mathrm{p}\left(\rho \sum_{i=1}^{n} y_{i}\right)\right]=\operatorname{e} \mathrm{p}\left(-\rho n \sqrt{\mathcal{E}_{k}}\right) \\
& \times \prod_{i=1}^{n}\left\{E\left[\mathrm{e} \mathrm{p}\left(\rho \tilde{v}_{i}\right)\right] \prod_{\substack{j=1 \\
j \neq k}}^{K} E\left[\mathrm{e} \mathrm{p}\left(\rho b_{i} \tilde{b}_{j} \sqrt{\mathcal{E}_{j}}\right)\right]\right\} .
\end{aligned}
$$

Because $\tilde{v}_{i}$ is conditionally Gaussian, $E\left[\tilde{v}_{i} \mid b_{i} \neq\right.$ $0]=0$, and $\operatorname{var}\left\{\tilde{v}_{i} \mid b_{i} \neq 0\right\}=\sigma_{v}^{2}$, where $\sigma_{v}^{2}$ is the AWGN variance. Therefore,

$$
E\left[\mathrm{e} p\left(\rho \tilde{v}_{i}\right)\right]=\operatorname{e} \mathrm{p}\left(\frac{\rho^{2} \sigma_{v}^{2}}{2}\right) .
$$

Using the pmf of $b_{i} \tilde{b}_{j}$ noted above, we find

$$
E\left[\operatorname{ep}\left(\rho b_{i} \tilde{b}_{j} \sqrt{\mathcal{E}_{j}}\right)\right]=(1-2 \alpha)+2 \alpha \cosh \left(\rho \sqrt{\mathcal{E}_{j}}\right) \text {. }
$$

To simplify the bound derivation, we note that $f(x)=(1-2 \alpha)+2 \alpha \cosh (x)$ is closely appro imated by e $\mathrm{p}\left(\alpha x^{2}\right)$, for $0<x \leq 1$, and $0<\alpha \leq 1 / 2$. We show subsequently that for cases of interest, $\rho \sqrt{\mathcal{E}_{j}}<1$. This appro imation may also be obtained by assuming the MUI has Gaussian distribution, and noting that $\operatorname{var}\left\{b_{i} \tilde{b}_{j} \sqrt{\mathcal{E}_{j}}\right\}=2 \alpha \mathcal{E}_{j}$, resulting in

$$
E\left[\mathrm{e} \mathrm{p}\left(\rho b_{i} \tilde{b}_{j} \sqrt{\mathcal{E}_{j}}\right)\right]=\mathrm{e} \mathrm{p}\left(\rho^{2} \alpha \mathcal{E}_{j}\right)
$$

which is very nearly equal to the actual e pectation in (23) for small $\rho \sqrt{\mathcal{E}_{j}}$.

Using (22) and (24) in (21) we obtain

$$
\begin{aligned}
& E\left[\operatorname{e} \mathrm{p}\left(\rho \sum_{i=1}^{n} y_{i}\right)\right]<\mathrm{e} \mathrm{p}\left(-\rho n \sqrt{\mathcal{E}_{k}}\right) \\
& \times \prod_{i=1}^{n}\left\{\operatorname{ep}\left(\frac{\rho^{2} \sigma_{v}^{2}}{2}\right) \prod_{\substack{j=1 \\
j \neq k}}^{K} \mathrm{e}\left(\rho^{2} \alpha \mathcal{E}_{j}\right)\right\} \\
& =\mathrm{e} \mathrm{p}\left(-\rho n \sqrt{\mathcal{E}_{k}}\right) \mathrm{e} \mathrm{p}\left(\frac{n \rho^{2} \sigma_{v}^{2}}{2}\right) \text { e } \mathrm{p}\left(n \rho^{2} \alpha \sum_{\substack{j=1 \\
j \neq k}}^{K} \mathcal{E}_{j}\right) .
\end{aligned}
$$

The value of $\rho>0$ that minimizes (20) may be found by minimizing the e ponential arguments in (25). We find

$$
\rho_{0}=\frac{\sqrt{\mathcal{E}_{k}}}{I_{0}}
$$




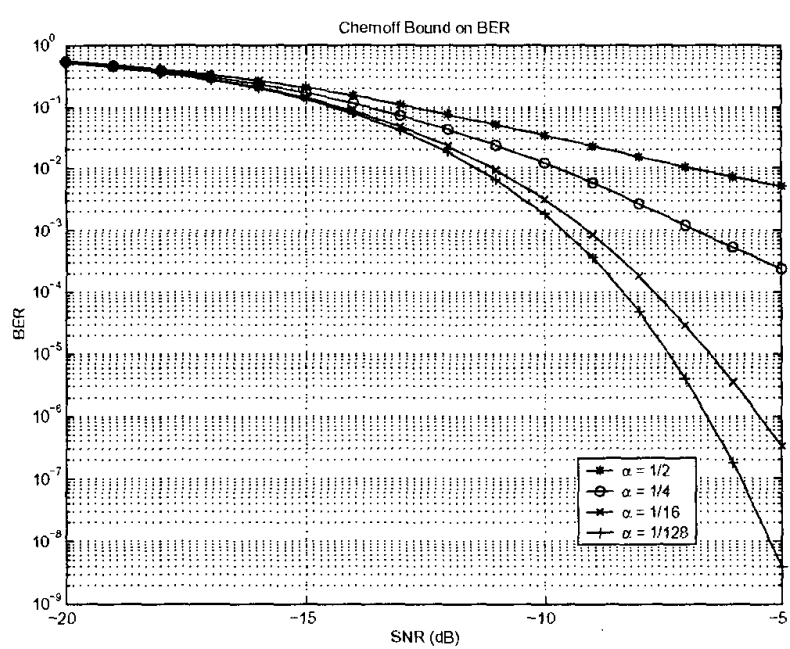

Figure 1: Chernoff bound on matched filter BER. All users have equal power, varying over $[-20,-5] \mathrm{dB}$ SNR.

where $I_{0}$ is the total interference given by

$$
I_{0}=\sigma_{v}^{2}+2 \alpha \sum_{\substack{j=1 \\ j \neq k}}^{K} \mathcal{E}_{j}
$$

The first term in $I_{0}$ is the AWGN variance, which contributes to all the non-zero correlation samples $y_{i}$. The second term is the total MUI energy for the $K-1$ undesired users, scaled by the probability of a collision between the desired user chip sequence $b_{i}$ and the interfering users chip sequences $\tilde{b}_{j}$. Recalling the appro imation to $(23)$, we find that

$$
\rho_{0} \sqrt{\mathcal{E}_{j}}=\frac{\sqrt{\mathcal{E}_{k} \mathcal{E}_{j}}}{I_{0}}
$$

which is generally small for cases of interest.

Substitution of $\rho_{0}$ into (25) provides the desired Chernoff bound,

$$
P_{e}=-\operatorname{Pr}(y>0 \mid-)<\operatorname{ep}\left(\frac{-n \mathcal{E}_{k}}{2 I_{0}}\right) .
$$

Thus we obtain a bound on the probability of bit error in AWGN with MUI, for an episodic binary DS signal integrating over $n$ pulses, e pressed in terms of the desired user energy $\mathcal{E}_{k}$, the interference energy $I_{0}$, and the probability of the chip sequences colliding given that $b_{i} \neq 0$, which is $2 \alpha$. We may call $2 \alpha$ the $M U I$ reduction factor, which is unity for non-episodic transmission (the conventional DS case). With all else fi ed, lowering $\alpha$ makes the pulses more episodic and correspondingly lowers the data rate, which then lowers

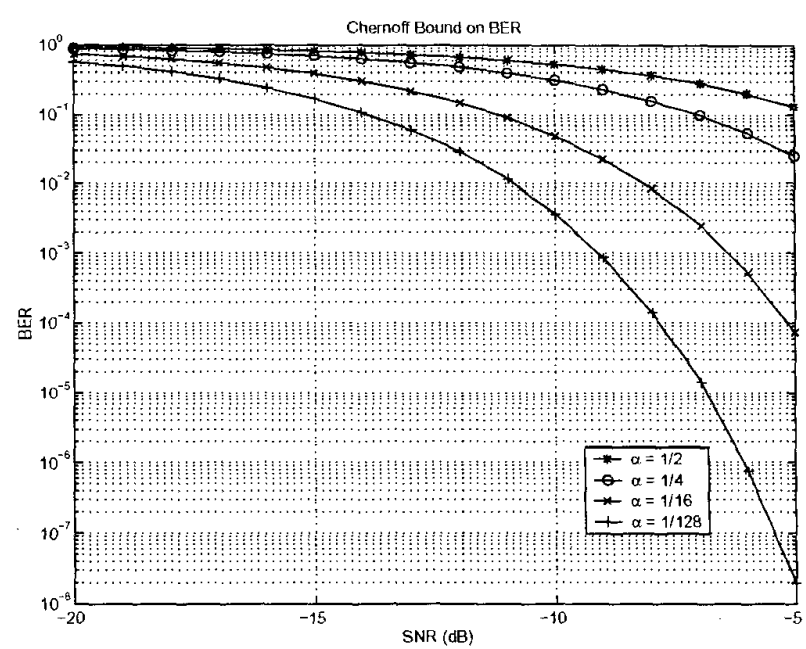

Figure 2: Chernoff bound on matched filter BER. Interfering users have $\mathrm{SNR}=0 \mathrm{~dB}$, while the desired user SNR is varied over $[-20,-5] \mathrm{dB}$.

the probability of pulse collision and hence the BER bound.

Example. We show (29) for two cases in Figures 1 and 2, with curves parameterized by $\alpha$. We have $K=10$ users, where SNR $=10 \log _{10}\left(\mathcal{E}_{j} / \sigma^{2}\right)$ is the signal-to-noise of the desired user with respect to the AWGN. In Figure 1, all users have the same SNR, so that at high SNR the desired user matched filter receiver performance is limited by MUI. However, as $\alpha$ becomes small the MUI may be significantly reduced. Figure 2 corresponds to a strong MUI case. Now, the undesired users are $f$ ed at $0 \mathrm{~dB}$ SNR, while the desired user SNR is varied from $-20:-5 \mathrm{~dB}$. Without episodic transmission $(\alpha=1 / 2)$ the performance is poor due to the high power MUI, whereas episodic transmission may be employed to reduce the MUI to very low levels.

Extensions. In subsequent work we consider effects of multipath channels, as well as channel estimation, the use of training, and impact on system performance. The addition of multi-user detection is also studied.

\section{REFERENCES}

[1] A. Swami, B. M. Sadler, J. Turner, "On the coexistence of ultra-wideband and narrowband radio systems," Proc. IEEE Military Communications Conference (MILCOM 2001), October 2001.

[2] J. G. Proakis, Digital Communications (McGraw-Hill, 2001).

[3] A. J. Viterbi, CDMA, Principles of Spread Spectrum (Addison Wesley, 1995). 